70. Scull, C. W., Bach, T. F., and Pemberton, Ralph (1939): Ann. Int. Med., xii. 1463-1472.

71. Shenwood, K. K., AND Hutchins, L. R. (1939): Northwest Med., XXxviii. 257-260.

72. Sherwood, K. K., and Thomson, Marian E. (1939): Journ. Amer. Dietet. Assoc., xv. 1-4.

73. Simmons, E. E., ANd Dunn, F. L. (1939): Arch. Phys. Therapy., Xx. 547-553.

74. Smith-Petersen, M. N. (1939): Journ. Bone and Joint Surg., xxi. 269-288.

75. Snyder, R. G., Traeger, Cornelios, and Kelly, LeMoyne (1939): Ann. Int. Med., xii. 1672-1681.

76. SpINk, W. W. (1939): Amer. Journ. Med. Sci., cxcviii. 35-39.

77. SpInk, W. W., AND Flink, E. B. (1939): Bull. Minnesota Med. Foundation, i. 26-29.

78. Steinberg, C. L., and Suter, Louise C. (1939): Arch. Int. Med., lxiv. 483-492.

79. Steinbrocker, Отto (1939): Ann. Int. Med., xii. 1917-1939.

80. SwaIm, L. T. (1939): Journ. Bone and Joint Surg., xxi. 983-991.

81. Swett, P. P. (1939): New York State Journ. Med., xxxix. 2125-2131.

82. SwIFT, H. F., AND Brown, T. M. (1939): Science, lxxxix. 271-272.

83. Tarsy, J. M. (1939): Indust. Med., viii. 186-193.

84. Taussig, Helen B. (1939): Journ. Pediat., xiv. 581-592.

85. Tegner, W. S. (1939): Ann. Rheumat. Dis., i. 249-303.

86. Thomas, Caroline B., and France, Richard (1939): Bull. Johns Hopkins Hosp., lxiv. 67-77.

87. Tobias, Norman (1939): Int. Clin., (S. 2), iii. 173-182.

88. Toumey, J. W., JR. (1939): Surg., Gynec. and Obst., lxviii. 1029-1037.

89. WaUgh, J. R., ANd Dawber, T. R. (1939): Amer. Journ. Syph., Gonor, and Ven. Dis., xxiii. 477-489.

90. Wright, L. T., AND Logan, MYra (1939): Arch. Surg., xxxix. 108-121.

\title{
A STUDY OF CERTAIN BLOOD TESTS WHICH REVEAL COLLOIDAL ABNORMALITIES IN RHEUMATIC CONDITIONS*
}

\author{
BY H. L. MILLES AND H. B. SALT
}

\section{INTRODUCTION}

IN chronic rheumatic conditions in which the blood is often characterised by a decreased suspension stability, there often occur alterations in the amounts, proportions, or nature of the proteins and perhaps other colloidal blood constituents. Discussions of these colloidal abnormalities have been made fre-

* Received for publication April 10, 1941. 
quently and have been summarised by $\operatorname{Race}^{1}$ and by other authorities. In general, it is concluded that the suspension stability depends in some way upon the colloidal state of the blood. More recent work has confirmed that in rheumatoid arthritis plasma globulins, especially euglobulin and fibrinogen, tend to rise while albumin tends to fall, ${ }^{2}$ and that a determination of the suspension stability is a simple way of appraising these changes in the blood. In a study of serum proteins in rheumatoid disease by Scull et al. ${ }^{3}$ it is especially remarked that both the actual levels of albumin and globulin, as well as their ratio, are to be remembered in considering abnormal colloidal systems in rheumatism. Ropes et al. ${ }^{4}$ have confirmed that there is no absolute correlation between the blood suspension stability and the concentration of any of the plasma protein fractions, and they conclude that variations in stability are due to variations in the colloidal state of the plasma as a whole. This conclusion admits possibilities that substances other than proteins may contribute to the colloidal equilibrium, and that the effects of qualitative changes in the proteins may be of great importance. Indeed, albumin and globulin may not be distinct entities separable by chemical precipitation, an opinion discussed with other aspects of the problem by Davison. ${ }^{5}$ It thus appears to be of less value to estimate the protein fractions in a rheumatic blood than to carry out the suspension stability test, in which the behaviour of the red cells furnishes an indication of the state of the colloidal system of which they form part.

Many other non-specific tests have been devised for the detection of abnormalities in the state of the blood in various diseases, notably tuberculosis and liver disorders. Though these are more concerned with the precipitability of the plasma colloids, they bear some similarity to the suspension stability test, especially in their interpretation. A study of certain of these tests has been made by applying them to blood samples, which were collected primarily for routine suspension stability tests on 140 unselected adult persons afflicted with rheumatism or allied disease.

\section{EXPERIMENTAL}

Four tests were carried out on each blood sample, the specimen consisting of about $11 \mathrm{ml}$. venous blood, of which $6 \mathrm{ml}$. were promptly oxalated with $12 \mathrm{mg}$. potassium oxalate in one tube 
and the remainder allowed to clot for serum in a second tube. The oxalated whole blood was used for determination of suspension stability (S.S.) and packed cell volume (P.C.V.), while the residual plasma was taken for the formol-gel test (F.G.T.). The serum was used for the calcium chloride heat coagulation test and the mercuric chloride flocculation test.

Suspension Stability Test (Erythrocyte Sedimentation Test).This was determined without delay (usually at once, never later than three hours after collection) in $5 \mathrm{ml}$. capacity graduated centrifuge tubes, using undiluted oxalated blood by the method of Collins et al. ${ }^{6}$ After two hours' settling, the blood was centrifuged for determination of packed cell volume, and the crude S.S. value at one hour was corrected on the basis of the P.C.V. reading by the formula:

Corrected S.S. $=$ Crude S.S. $+f(42-$ P.C.V. $)$, where factor $f=1.5$ if P.C.V. is below 42 per cent., or 1.0 if P.C.V. is above 42 per cent., taking proper account of algebraic signs.

The S.S. test was regarded as a standard method of assessing the colloidal condition of the blood samples and results of the other tests were therefore related to these. Previous experience with the S.S. test had proved that healthy blood usually shows a stability of 90 per cent. or over, though sometimes it may be as low as 85 per cent. With very few exceptions, the most unstable bloods yield a minimum corrected stability value of 55 per cent., the lowest value ever obtained being 53 per cent. It must be noted that these values are one-hour corrected S.S. figures; crude S.S. values are found to spread over a wider range owing to the effects of varying cell volumes.

It was decided to divide the results into three arbitrary groups of almost equal range, namely, corrected S.S. 100 to 85, 84 to 70 , and 69 to 53 . The blood samples were unselected, and the results were placed into these three groups as they were obtained. However, an inspection of the accumulating results showed most features of interest in the low S.S. group, so that after thirty blood specimens had been tested in the high S.S. group and thirty in the medium S.S. group, only bloods of low suspension stability were continued with, until eighty results were available in the low S.S. group. Later this last group was subdivided into almost equal halves, separating thirty-eight blood specimens where the corrected S.S. lay between sixty-nine and sixty, from forty-two blood specimens where the range was 
fifty-nine to fifty-three. This subdivision served to mark off clearly the group of exceptionally unstable bloods.

The Formol Gel Test (Aldehyde Reaction).-This test, which depends upon the formation of a gel when formaldehyde is added to plasma, has been studied in chronic rheumatism by Gibson and Richardson, ${ }^{7}$ and in acute rheumatism by Green et al. ${ }^{8}$ The technique of these authors was adopted, and the test applied to the clear plasma of each blood sample after the suspension stability had been determined and the blood centrifuged at 3,000 r.p.m. for fifteen minutes. The arrangement of the test and method of recording the results is indicated in the following table:

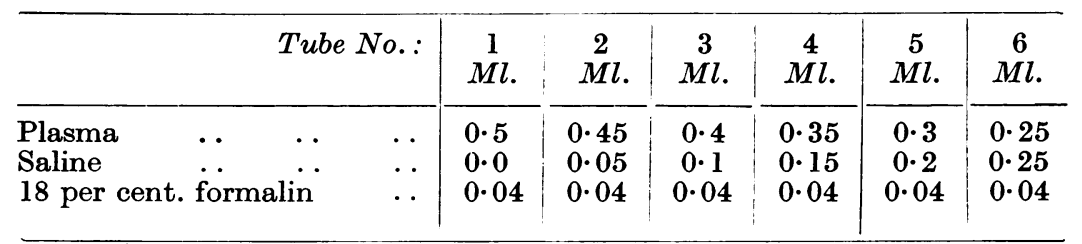

Mix, leave eighteen hours at room temperature, examine for gel formation.

Results : No gel=0. Partial gelation in tube $1=1$-plus or 2-plus. Complete gelation in tube $1=3$-plus, in tube $2=4$-plus, in tube $3=5$-plus, etc., up to 8-plus.

The formol-gel test, like the suspension stability test, is not specific for any pathological condition; in fact, positive reactions of the plasma are likely in any disease with an elevated plasma globulin, and the F.G.T. merely gives an indication of the colloidal state of the blood.

A good degree of correlation between the formol-gel reaction and the suspension stability of the blood has been obtained by both the authors quoted above. In the present instance, the F.G.T. was adopted as an accessory to the S.S. test, for comparison with the other tests described below and as a means of detecting anomalous S.S. results if they occurred.

The Calcium Chloride Heat Coagulation Test (Weltmann Reaction).-The Weltmann serum coagulation reaction ${ }^{9}$ has been studied in many diseases, amongst which are rheumatic conditions. ${ }^{10,11}$ In America, Levinson and co-workers have made a close study of the reaction in tuberculosis, rheumatic fever and other diseases, and valuable discussions are given in their publications, ${ }^{12}$. 13 14. 15 together with a description of the technique, 
especially well recorded with photographs of the reaction results in their paper of 1939. Essentially the test is as follows: From a stock solution of hydrated calcium chloride, 10 per cent. $\mathrm{CaCl}_{2}, 6 \mathrm{H}_{2} \mathrm{O}$, ten dilutions consisting of 0.1 per cent., 0.09 per cent., 0.08 per cent., 0.07 per cent., 0.06 per cent., 0.05 per cent., 0.04 per cent., 0.03 per cent., 0.02 per cent., and 0.01 per cent., are prepared freshly each day and numbered one to ten, beginning with the strongest. (This arbitrary numbering is quite unnecessary, but has been adopted so far by previous workers. The present authors have retained the numbering system, but would urge that the calcium chloride concentrations themselves are adequate designations, especially if expressed as decigrams per litre to keep in whole numbers. The order of such numbering, however, would be the reverse of the arbitrary numbering actually adopted.) Five ml. of each dilution are placed respectively in test tubes numbered one to ten, followed by $0.1 \mathrm{ml}$. unhæmolysed serum. After well mixing, the tubes are placed in a boiling-water bath for fifteen minutes, then removed and examined for coagulation, which is usually very easily distinguished from turbidity. The number of tubes in which coagulation occurs is called the serum coagulation band (S.C.B.). Healthy serum gives an S.C.B. of six; or there might be very slight coagulation also in tube number seven, which is recorded as S.C.B. $6 \frac{1}{2}$. Abnormal sera may give values above or below this figure. In order to preserve a uniform terminology, the descriptive title " calcium chloride heat coagulation test" is adopted here. The test was chosen for study because others have already obtained interesting results in rheumatic conditions, although the coagulation band has not always been found to parallel the suspension stability. The mechanism of the reaction is obscure, though obviously different results must depend on differences in the coagulable colloids of the serum.

The Mercuric Chloride Flocculation Test (Takata-Ara Reaction).-This reaction, first described by Takata and Ara in 1925, and afterwards much studied in Germany as a test for liver disease, became the subject of investigations by several workers in America. Crane ${ }^{16}$ reviewed its history, modified the technique and studied liver disease. Kirk ${ }^{17}$ reviewed all the previous results in the literature and contributed his own experiences. Bowman and Bray ${ }^{18}$ emphasised the non-specific nature of the reaction and its dependence on the amounts or proportions of 
the serum proteins. The history of the test was again reviewed by Chasnoff and Solomon ${ }^{19}$ and their method of carrying out the reaction and interpreting the results was carefully described. In the present study this method was adopted, which is essentially as follows:

One ml. 0.9 per cent. sodium chloride is placed in each of six small tubes. To the first tube, $1.0 \mathrm{ml}$. serum is added and mixed; $1.0 \mathrm{ml}$. of the mixture is then removed and added to the saline in the second tube; this procedure is continued throughout the six tubes, $1.0 \mathrm{ml}$. from the last tube being discarded, leaving serum dilutions of $1: 2$ to $1: 64$. To each is added $0 \cdot 25 \mathrm{ml}$. 10 per cent. sodium carbonate, followed, after mixing, by $0 \cdot 15$ ml. 0.5 per cent. mercuric chloride. All the tubes are then thoroughly shaken and set aside at room temperature for twèntyfour hours, when a pearly flocculum may have developed, indicating a positive reaction. Any other deposit is disregarded. If the flocculation is definite in four or more tubes, a strong positive (3-plus) result is indicated; if definite but slight in three or four tubes, a positive (2-plus) result; if definite but minimal in three tubes, a weak positive (1-plus) result; all other results are negative. The method of interpreting the results is important, as different investigators have applied different criteria for evaluation of the findings. The 1-plus positive results here recorded were almost always indicated by the last three tubes showing the flocculum.

It is emphasised by Chasnoff and Solomon that the mercuric chloride reaction is not specific for liver disease, and that it is likely to be positive whenever the albumin-globulin ratio in the serum is decreased. This is the most important, though not the sole factor, in the mechanism of the reaction; a conclusion supported by the results of Taran and Lipstein, ${ }^{20}$ who studied the test in tuberculous patients. Hitherto the test does not seem to have been applied to the blood of rheumatic patients, but its trial is justified by the essentially non-specific nature of the test and the dependence of the results upon the colloidal state of the serum. Positive results have been obtained in active tuberculosis where low suspension stabilities are found, and in kala-azar, where the formol gel reactions are characteristically intense, though the greatest incidence of positive mercuric chloride tests is to be found in hepatic cirrhosis. 


\section{Discussion of Results}

The experimental findings are recorded in Tables I, II, IIIA $\frac{\mathrm{O}}{\omega}$. and ІІІв, arranged in order of decreasing suspension stability

Table I.-Thirty Cases with Corrected S.S. in the Range 101 to 85.

\begin{tabular}{|c|c|c|c|c|c|c|c|}
\hline $\begin{array}{l}\text { Case } \\
\text { No. } \\
\text { and } \\
\text { Sex. }\end{array}$ & Diagnosis, etc. & $\begin{array}{c}\text { Crude } \\
\text { S.S., } \\
1 \text { Hour. }\end{array}$ & $\begin{array}{c}\text { Cor- } \\
\text { rected } \\
\text { S.S., } \\
1 \text { Hour. }\end{array}$ & $\begin{array}{c}\text { P.C.V. } \\
\text { per } \\
\text { Cent. }\end{array}$ & $\begin{array}{c}\text { Formol } \\
\text { Gel } \\
\text { Test. }\end{array}$ & $\begin{array}{c}\text { Serum } \\
\text { Coagu- } \\
\text { lation } \\
\text { Band. }\end{array}$ & $\begin{array}{l}\text { Mercuric } \\
\text { Chloride } \\
\text { Floccula- } \\
\quad \text { tion. }\end{array}$ \\
\hline $185, \mathrm{~F}$. & R.A., Group 2 & 90 & 101 & 35 & 0 & $7 \frac{1}{2}$ & 0 \\
\hline $198, \mathrm{~F}$ & (?) R.A. & 77 & 100 & 27 & 0 & $7^{2}$ & 0 \\
\hline $104, \mathrm{M}$ & Fibrositis & 97 & 97 & 42 & 0 & 6 & 0 \\
\hline $132, \mathrm{~F}$ & (?) R.A. & 96 & 96 & 42 & 0 & $6 \frac{1}{2}$ & 0 \\
\hline $123, \mathrm{~F}$. & Fibrositis & 92 & 95 & 40 & 0 & 6 & 0 \\
\hline $125, \mathrm{~F}$ & Osteo-arthritis & 89 & 95 & 38 & 0 & 6 & 0 \\
\hline $66, \mathrm{M}$ & (?) Strain & 92 & 94 & 41 & 0 & 6 & 0 \\
\hline $186, \mathrm{M}$. & $\begin{array}{l}\text { Fibrositis, peri-articu- } \\
\text { lar }\end{array}$ & 96 & 94 & 44 & 0 & $8 \frac{1}{2}$ & 0 \\
\hline $181, \mathrm{~F}$ & R.A., Group 1 & 94 & 93 & 43 & 0 & $7 \frac{1}{2}$ & 0 \\
\hline $87, \mathrm{M}$ & R.A., Group 1 & 99 & 93 & 48 & 0 & 6 & 0 \\
\hline $92, \mathrm{M}$ & $\begin{array}{l}\text { Fibrositis, peri-articu- } \\
\text { lar }\end{array}$ & 95 & 92 & $4 \tilde{5}$ & 0 & 6 & 0 \\
\hline i26, M. & Lumbago, joint pain & 96 & 92 & 46 & 0 & 7 & 0 \\
\hline 127, M. & (?) R.A. & 95 & 92 & 45 & $\mathbf{0}$ & 6 & $\mathbf{0}$ \\
\hline $83, \mathrm{M}$. & Fibrositis & 95 & 91 & 46 & 0 & $6 \frac{1}{2}$ & 0 \\
\hline $70, \mathrm{~F}$ & (?) pre-R.A. & 92 & 91 & 43 & 0 & $6 \frac{1}{2}$ & 0 \\
\hline $137, \mathrm{M}$. & (?) R.A. & 90 & 90 & 42 & 0 & 6 & 0 \\
\hline $175, \mathrm{M}$ & R.A. (definite) & 84 & 90 & 38 & 0 & 8 & 0 \\
\hline $94, \mathrm{~F}$ & Fibrositis & 80 & 89 & 36 & 0 & $6 \frac{1}{2}$ & 0 \\
\hline $152, \mathrm{M}$. & R.A., Group 2 & 91 & 89 & 44 & 0 & 7 & 0 \\
\hline $121, \mathrm{~F}$ & Fibrositis & 89 & 88 & 43 & 0 & 5 & 0 \\
\hline 133, F. & Fibrositis & 88 & 88 & 42 & 0 & 6 & 0 \\
\hline $134, \mathrm{M}$ & R.A., Group 2 & 90 & 88 & 44 & 0 & $7 \frac{1}{2}$ & $\mathbf{0}$ \\
\hline $162, \mathrm{M}$. & Fibrositis & 85 & 88 & 40 & 0 & 7 & 0 \\
\hline 164, M. & Chronic gout & 93 & 88 & 47 & 0 & 7 & 0 \\
\hline 75, M. & Fibrositis & 82 & 88 & 38 & 0 & 5 & 0 \\
\hline $89, \mathrm{M}$. & Erythema nodosum & 90 & 88 & 44 & 0 & $6 \frac{1}{2}$ & 0 \\
\hline 47, M. & Gout & 93 & 87 & 48 & 0 & $6 \frac{1}{2}$ & 0 \\
\hline $59, \mathrm{~F}$. & R.A., Group 1 & 80 & 86 & 38 & 0 & $5 \frac{1}{2}$ & 0 \\
\hline 91, M. & Synovitis & 92 & 86 & 48 & 0 & $5 \frac{1}{2}$ & 0 \\
\hline $81, \mathrm{~F}$. & Osteo-arthritis & 78 & 86 & 37 & 0 & 6 & 0 \\
\hline
\end{tabular}

and divided into groups corresponding to corrected S.S. values of 101 to 85,84 to 70,69 to 60 , and 59 to 53 . The first two cases of Table I are examples of anæmia in the absence of diminished suspension stability, a phenomenon discussed by Collins et $a l .{ }^{6}$ which explains the apparently absurd values of 100 or over for the corrected S.S. In every other respect the corrected S.S. values are regiarded as the most satisfactory characteristics of the blood samples upon which to base a grouping and comparison of results.

Comparing the formol-gel test with suspension stabilities, it 
is immediately apparent that with only one exception (partial gelation) the F.G.T. is negative whenever the corrected S.S. is above seventy (fifty-nine cases out of sixty). Where the corrected S.S. is below seventy, the F.G.T. is usually positive, the incidence being seventy-three times out of eighty. The seven

Table II.-Thirty Cases with Corrected S.S. in the Range 84 to 70.

\begin{tabular}{|c|c|c|c|c|c|c|c|}
\hline $\begin{array}{l}\text { Case } \\
\text { No. } \\
\text { and } \\
\text { Sex. }\end{array}$ & Diagnosis, etc. & $\begin{array}{l}\text { Crude } \\
\text { S.S., } \\
1 \text { Hour. }\end{array}$ & \begin{tabular}{|c|} 
Cor- \\
rected \\
S.S., \\
1 Hour.
\end{tabular} & $\begin{array}{c}\text { P.C.V. } \\
\text { per } \\
\text { Cent. }\end{array}$ & $\begin{array}{c}\text { Formol } \\
\text { Gel } \\
\text { Test. }\end{array}$ & $\begin{array}{l}\text { Serum } \\
\text { Coagu- } \\
\text { lation } \\
\text { Band. }\end{array}$ & $\begin{array}{l}\text { Mercuric } \\
\text { Chloride } \\
\text { Floccula- } \\
\quad \text { tion. }\end{array}$ \\
\hline $84, \mathrm{~F}$. & R.A. Group 1 & 81 & 84 & 40 & $\mathbf{0}$ & $6 \frac{1}{2}$ & $\mathbf{0}$ \\
\hline 179, M. & Gout, synovitis & 84 & 84 & 42 & $\mathbf{0}$ & $7^{2}$ & 0 \\
\hline $160, \mathrm{~F}$. & Fibrositis & 80 & 83 & 40 & 0 & $6 \frac{1}{2}$ & $\mathbf{0}$ \\
\hline $153, \mathrm{~F}$. & R.A., Group 2 & 80 & 83 & 40 & 0 & $6 \frac{1}{2}$ & 0 \\
\hline $72, \mathrm{~F}$. & Fibrositis & 75 & 83 & 37 & 0 & $7^{2}$ & 0 \\
\hline $158, \mathrm{M}$. & R.A., Group 2 & 85 & 82 & 45 & 0 & $6 \frac{1}{2}$ & 0 \\
\hline $195, \mathrm{~F}$. & R.A., Group 2 & 71 & 82 & 35 & 0 & 8 & 0 \\
\hline $111, \mathrm{~F}$. & Fibrositis & 73 & 82 & 36 & 0 & 6 & 0 \\
\hline $112, \mathrm{~F}$. & $\begin{array}{l}\text { Osteo and rheumatoid } \\
\text { arthritis }\end{array}$ & 72 & 81 & 36 & 0 & 5 & 0 \\
\hline $172, F$ & R.A., Group 1 & 81 & 81 & 42 & 0 & 7 & $\mathbf{0}$ \\
\hline $146, \mathrm{M}$. & R.A., Group 3 & 80 & 80 & 42 & 0 & $4 \frac{1}{2}$ & 0 \\
\hline $62, \mathrm{~F}$ & (?) R.A. & $\mathbf{7 7}$ & 79 & 41 & 0 & $4 \frac{1}{2}$ & 0 \\
\hline $86, \mathrm{M}$. & Fibrositis, strain & 81 & 79 & 44 & 2 -plus & $6^{2}$ & 0 \\
\hline $122, \mathrm{~F}$. & R.A., Group 2 & 71 & 79 & 37 & 0 & 6 & 0 \\
\hline $167, \mathrm{M}$. & R.A., Group 2 & 81 & 79 & 44 & 0 & 7 & $\mathbf{0}$ \\
\hline $155, \mathrm{~F}$. & R.A., Group 2 & 78 & 78 & 42 & 0 & $7 \frac{1}{2}$ & $\mathbf{0}$ \\
\hline 197, F. & R.A., Group 2 & 72 & 78 & 38 & 0 & $7^{2}$ & $\mathbf{0}$ \\
\hline $144, F$. & Fibrositis & 79 & 76 & 45 & 0 & $5 \frac{1}{2}$ & $\mathbf{0}$ \\
\hline I51, M. & $\begin{array}{l}\text { Rheumatic fever } \\
\text { (mild) }\end{array}$ & 80 & 76 & 46 & 0 & $6 \frac{1}{2}$ & 0 \\
\hline 58, M. & R.A., Group 1 & 80 & 75 & 47 & 0 & $6 \frac{1}{2}$ & $\mathbf{0}$ \\
\hline $76, F$ & Fibrositis & 75 & 75 & 42 & 0 & $5 \frac{1}{2}$ & $\mathbf{0}$ \\
\hline $165, M$. & Chronic gout & 72 & 75 & 40 & 0 & $7^{2}$ & $\mathbf{0}$ \\
\hline 191, F. & R.A., Group 1 & 73 & 75 & 41 & $\mathbf{0}$ & 7 & $\mathbf{0}$ \\
\hline $192, \mathrm{~F}$. & R.A., Group 2 & 59 & 74 & 32 & 0 & $6 \frac{1}{2}$ & 0 \\
\hline $145, \mathrm{M}$. & R.A., Group 2 & 76 & 73 & 45 & $\mathbf{0}$ & 5 & $\mathbf{0}$ \\
\hline 61, M. & R.A., Group 2 & 67 & 72 & 39 & $\mathbf{0}$ & $4 \frac{1}{2}$ & 0 \\
\hline $103, F$ & R.A. (severe) & 72 & 72 & 42 & 0 & $4 \frac{1}{2}$ & 0 \\
\hline $163, \mathrm{M}$. & Spondylitis ankylosis & 75 & 72 & 45 & 0 & 5 & 0 \\
\hline 147, F. & R.A., Group 2 & 70 & 70 & 42 & 0 & 6 & 0 \\
\hline 178, M. & R.A., Group 1 & 67 & 70 & 40 & 0 & $7 \frac{1}{2}$ & 0 \\
\hline
\end{tabular}

instances of negative formol-gel reactions in bloods of corrected S.S. less than seventy are discussed later. A comparison of corrected S.S. and F.G.T. results is summarised in Table IV on page 38.

In general, the correlation between the two tests found by Gibson and Richardson ${ }^{7}$ and by Green et al. ${ }^{8}$ is confirmed, the present results being even more clear cut, probably because corrected S.S. values are adopted instead of crude S.S. values. It is apparent that the F.G.T. is less sensitive than the S.S. test, 
but that it can be especially useful in further characterising those bloods whose suspension stabilities lie in the lowest range.

The correlation existing between the F.G.T. and anæmia is

Table IIIA.-Thirty-Eight Cases with Corrected S.S. in the Range 69 то 60.

\begin{tabular}{|c|c|c|c|c|c|c|c|}
\hline $\begin{array}{l}\text { Case } \\
\text { No. } \\
\text { and } \\
\text { Sex. }\end{array}$ & Diagnosis, etc. & $\begin{array}{c}\text { Crude } \\
\text { S.S., } \\
1 \text { Hour. }\end{array}$ & $\begin{array}{c}\text { Cor- } \\
\text { rected } \\
\text { S.S., } \\
1 \text { Hour. }\end{array}$ & $\begin{array}{c}\text { P.C.V. } \\
\text { per } \\
\text { Cent. }\end{array}$ & $\begin{array}{c}\text { Formo } \\
\text { Gel } \\
\text { Test. }\end{array}$ & $\begin{array}{l}\text { Serum } \\
\text { Coagu- } \\
\text { lation } \\
\text { Band. }\end{array}$ & $\begin{array}{l}\text { Mercuri } \\
\text { Chloride } \\
\text { Floccula } \\
\text { tion. }\end{array}$ \\
\hline $120, \mathrm{~F}$. & Fibrositis & 70 & 69 & 43 & 0 & 6 & 0 \\
\hline $50, \mathrm{M}$. & R.A., (three months) & 69 & 69 & 42 & & $5 \frac{1}{2}$ & 1-plus \\
\hline 60, M. & R.A., Group 1 & 73 & 69 & 46 & 1-plus & $6 \frac{1}{2}$ & \\
\hline $105, \mathrm{~F}$. & Rheumatoid arthritis & 57 & 69 & 34 & 3 & 5 & 1-plus \\
\hline $210, \mathrm{M}$. & R.A., Group 2 & 69 & 69 & 42 & 3, & $6 \frac{1}{2}$ & \\
\hline $138, \mathrm{~F}$. & R.A., Group 3 & 59 & 68 & 36 & 3, & $4 \frac{1}{2}$ & \\
\hline $99, \mathrm{~F}$ & Rheumatoid arthritis & 61 & 67 & 38 & 3, & 6 & 1-plus \\
\hline $129, \mathrm{~F}$. & R.A., Group 1 & 64 & 67 & 40 & 3, & 7 & \\
\hline $154, \mathrm{~F}$. & (?) R.A., (six months) & 67 & 67 & 42 & 0 & 6 & \\
\hline $77, \mathrm{M}$. & Osteo-arthritis & 61 & 66 & 39 & & 6 & 1-plus \\
\hline $166, \mathrm{M}$. & $\begin{array}{l}\text { R.A., Group } 2 \text { (five } \\
\text { months) }\end{array}$ & 60 & 66 & 38 & & $7 \frac{1}{2}$ & \\
\hline 106, M. & Fibrositis & 71 & 65 & 48 & & $5 \frac{1}{2}$ & 2-plus \\
\hline $109, \mathrm{M}$. & R.A., Group 3 & 71 & 65 & 48 & & 6 & \\
\hline $110, \mathrm{M}$. & R.A., Group 3 & 66 & 65 & 43 & 3, & $5 \frac{1}{2}$ & 0 \\
\hline $131, \mathrm{~F}$. & $\begin{array}{l}\text { R.A. (no symptoms } \\
\text { for two years) }\end{array}$ & 57 & 67 & 37 & 0 & 6 & 0 \\
\hline $135, \mathrm{M}$. & R.A., Group 2 & 68 & 65 & 45 & 2, & $4 \frac{1}{2}$ & 0 \\
\hline $161, \mathrm{~F}$. & R.A., Group 3 & 45 & 65 & 29 & 6, & $6 \frac{1}{2}$ & 0 \\
\hline $80, \mathrm{~F}$. & R.A., Group 2 & 61 & 64 & 40 & 3, & 4 & \\
\hline $101, \mathrm{~F}$. & Fibrositis & 65 & 64 & 43 & 3, & $6 \frac{1}{2}$ & 1-plus \\
\hline $177, \mathrm{M}$. & R.A., Group 3 & 65 & 64 & 43 & 3 & 8 & 0 \\
\hline $115, \mathrm{M}$. & $\begin{array}{l}\text { Spondylitis ankylosis } \\
\text { (early) }\end{array}$ & 61 & 63 & 41 & 3 & $4 \frac{1}{2}$ & 0 \\
\hline 184, M. & R.A., Group 1 . & 66 & 63 & 45 & 0 & $6 \frac{1}{2}$ & 0 \\
\hline $96, \mathrm{~F}$ & Rheumatoid arthritis & 64 & 62 & 44 & 3, & 6 & 0 \\
\hline $102, \mathrm{M}$. & $\begin{array}{l}\text { Meningococcal septi- } \\
\text { cæmia }\end{array}$ & 62 & 62 & 42 & 5, & $1 \frac{1}{2}$ & 0 \\
\hline $114, \mathrm{M}$. & R.A., Group 3 & 59 & 62 & 40 & 2, & 6 & 2-plus \\
\hline $119, \mathrm{~F}$. & Gonococcal arthritis & 51 & 62 & 35 & 4, & 7 & \\
\hline $173, \mathrm{M}$. & R.A., Group 2 & 64 & 62 & 44 & 3, & $6 \frac{1}{2}$ & 0 \\
\hline $180, \mathrm{M}$. & R.A., Group 3 & 64 & 62 & 44 & 4, & $6 \frac{1}{2}$ & \\
\hline $56, \mathrm{~F}$ & Muscular stiffness & 52 & 61 & 36 & $4 "$ & $6 \frac{1}{2}$ & 1-plus \\
\hline $65, \mathrm{~F}$. & R.A., Group 2 & 55 & 61 & 38 & 3, & $5 \frac{1}{2}$ & 0 \\
\hline $140, \mathrm{M}$. & R.A., Group 3 & 61 & 61 & 42 & $4 "$ & $5 \frac{1}{2}$ & 0 \\
\hline $143, \mathrm{~F}$. & $\begin{array}{l}\text { R.A., Group } 3 \text { (fifteen } \\
\text { years) }\end{array}$ & 50 & 61 & 35 & 0 & 4 & 0 \\
\hline $168, \mathrm{M}$. & R.A., Group 3 & 38 & 61 & 27 & 4, & $5 \frac{1}{2}$ & 0 \\
\hline $183, \mathrm{M}$. & R.A., Group 3 & 63 & 61 & 44 & 3, & $5 \frac{1}{2}$ & \\
\hline $98, \mathrm{~F}$ & Rheumatoid arthritis & 45 & 60 & 32 & 4, & 4 & 1-plus \\
\hline $100, \mathrm{M}$. & Rheumatoid arthritis & 57 & 60 & 40 & 4, & 5 & \\
\hline $170, \mathrm{~F}$. & R.A., Group 3 & 49 & 60 & 35 & 7, & 7 & 1-plus \\
\hline $171, \mathrm{M}$. & R.A., Group 2 & 60 & 60 & 42 & 2, & 7 & 0 \\
\hline
\end{tabular}

also confirmed by present results. Table $\mathrm{V}$ on page 38 shows the $\widetilde{N}$ relationship between the degree of positivity of the formol-gel reactions and the average packed cell volumes.

As the formol-gel reactions become more intense, the average 
P.C.V. falls, as does the average crude S.S., but the phenomenon is apparently unrelated to the true or corrected S.S., which is fairly constant throughout the range of formol-gel results. On

Tabie IIIb.-Forty-Two Cases with Corrected S.S. in the Range 59 то 53.

\begin{tabular}{|c|c|c|c|c|c|c|c|}
\hline $\begin{array}{l}\text { Case } \\
\text { No. } \\
\text { and } \\
\text { Sex }\end{array}$ & Diagnosis, etc. & $\begin{array}{c}\text { Crude } \\
\text { S.S., } \\
1 \text { Hour }\end{array}$ & $\begin{array}{c}\text { Cor- } \\
\text { rected } \\
\text { S.S., } \\
1 \text { Hour. }\end{array}$ & $\begin{array}{c}\text { P.C.V. } \\
\text { per } \\
\text { Cent. }\end{array}$ & $\begin{array}{c}\text { Formol } \\
\text { Gel } \\
\text { Test. }\end{array}$ & $\begin{array}{l}\text { Serum } \\
\text { Coagu- } \\
\text { lation } \\
\text { Band. }\end{array}$ & $\begin{array}{c}\text { Mercuric } \\
\text { Chloride } \\
\text { Floccula- } \\
\text { tion. }\end{array}$ \\
\hline $44, \mathrm{~F}$. & Fibrositis & 51 & 59 & 37 & 3-plus & 5 & 0 \\
\hline 53, M. & R.A. (severe) & 47 & 59 & 34 & 5 & 3 & 0 \\
\hline 93, F. & R.A., Group 3 & 42 & 59 & 31 & 7, & $5 \frac{1}{2}$ & 2-plus \\
\hline $128, \mathrm{M}$. & Gout & 59 & 59 & 42 & 3 " & 5 & \\
\hline $\begin{array}{l}\text { 136, F. } \\
74, \mathrm{~F}\end{array}$ & & 57 & 59 & 41 & 1, & 5 & 0 \\
\hline $74, \mathrm{~F}$. & Fibrositis (R.A.) & 49 & 58 & 36 & 4 & 3 & 0 \\
\hline $113, \mathrm{~F}$. & R.A., Group 3 & 44 & 58 & 33 & 8 & 4 & 3-plu \\
\hline $118, \mathrm{M}$. & R.A., Group 2 & 53 & 58 & 39 & 4, & 6 & $1 "$ \\
\hline $124, F$. & Rheumatoid arthritis & 58 & 58 & 42 & 3, & 6 & 0 \\
\hline $141, \mathrm{~F}$. & R.A., Group 3 & 46 & 58 & 34 & 6 & 5 & 0 \\
\hline $157, \mathrm{M}$. & R.A., Group & 52 & 58 & 38 & 3, & $6 \frac{1}{2}$ & 0 \\
\hline $174, \mathrm{~F}$. & Rheumatoid arthritis & 58 & 58 & 42 & 3 & $7 \frac{1}{2}$ & 0 \\
\hline $182, \mathrm{M}$. & R.A., Group 3 & 58 & 58 & 42 & 5, & $4 \frac{1}{2}$ & 0 \\
\hline $199, \mathrm{~F}$. & R.A., Group 3 & 43 & 58 & 32 & $4 "$ & $5 \frac{1}{2}$ & 0 \\
\hline $204, F$. & Rheumatoid arthritis & 52 & 58 & 38 & 3 & $6 \frac{1}{2}$ & 0 \\
\hline $46, \mathrm{M}$. & Rheumatoid arthritis & 55 & 57 & 41 & 3 & $7^{2}$ & 0 \\
\hline $142, \mathrm{~F}$. & & 46 & 57 & 35 & 5, & $4 \frac{1}{2}$ & 0 \\
\hline $156, \mathrm{~F}$. & Gout & 39 & 57 & 30 & 3 & 5 & 0 \\
\hline $169, \mathrm{M}$. & R.A., Group 3 & 55 & 57 & 41 & 3 & 7 & 0 \\
\hline $190, \mathrm{M}$. & Spondylitis, ankylosing & 48 & 57 & 36 & $5 "$ & 6 & 1-plus \\
\hline $194, \mathrm{M}$. & R.A., Group 3 & 43 & $\mathbf{5 7}$ & 33 & 4 & $5 \frac{1}{2}$ & 0 \\
\hline $207, \mathrm{~F}$. & R.A., Group 3 & 51 & 57 & 38 & 4 & $5 \frac{1}{2}$ & 0 \\
\hline 73, M. & Osteo-arthritis (early) & 61 & 56 & 47 & 3 & 5 & 0 \\
\hline $90, \mathrm{M}$. & Gout & 56 & 56 & 42 & 3 & $5 \frac{1}{2}$ & 0 \\
\hline $117, \mathrm{~F}$. & Rheumatoid arthritis & 54 & 56 & 41 & 3 & $6 \frac{1}{2}$ & 0 \\
\hline $130, \mathrm{~F}$. & R.A., Group & 50 & 56 & 38 & 3 & $5 \frac{1}{2}$ & 0 \\
\hline $149, \mathrm{~F}$. & R.A., Group 2 & 48 & 56 & 37 & $4 "$ & $5^{2}$ & 0 \\
\hline $150, M$. & R.A., Group 3 & 54 & 56 & 41 & 3 & 5 & 0 \\
\hline $159, \mathrm{~N}$ & R.A., Group & 57 & 56 & 43 & $4 "$, & 7 & 0 \\
\hline 187, M. & Rheumatoid arthritis & 50 & 56 & 38 & $6 "$, & $5 \frac{1}{2}$ & 0 \\
\hline 188, M. & R.A., Grou & 56 & 56 & 42 & 3 & $6 \frac{1}{2}$ & 0 \\
\hline $196, N$ & R.A., Grou & 41 & 56 & 32 & 5 & $7^{2}$ & 1-plus \\
\hline $202, F$ & R.A., Group 2 & 47 & 56 & 36 & 4, & 6 & 0 \\
\hline $57, \mathrm{~F}$. & R.A., Group 3 & 55 & 55 & 42 & 3 & $5 \frac{1}{2}$ & 1-plus \\
\hline 148, M. & R.A., Group & 53 & 55 & 41 & $4 "$ & 5 & 0 \\
\hline $189, N$ & Rheumatoid arthritis & 47 & 5 & 3 & 4 & 6 & 0 \\
\hline $200, N$ & R.A., Group 3 & 47 & 5 & 37 & 4 & 5 & 0 \\
\hline 201,1 & R.A., Grou & 44 & 55 & 35 & 5 & 3 & 0 \\
\hline $203, F$. & R.A., Group 3 & 43 & 54 & 35 & $5 "$ & 6 & 0 \\
\hline $193, \mathrm{~F}$. & R.A., Group 3 & 50 & 53 & 40 & $4 "$, & $6 \frac{1}{2}$ & 0 \\
\hline $88, \mathrm{M}$. & Acute rheumatic fever & 50 & 53 & 40 & 5 & $2 \frac{1}{2}$ & 0 \\
\hline $116, \mathrm{M}$. & Acute rheumatic fever & 48 & 53 & 39 & 4 & 0 & 0 \\
\hline
\end{tabular}

the other hand, many anæmic bloods occur in Tables I and II, where the F.G.T. is quite negative and the S.S. ranges from normal to seventy, showing that a low S.S. is a necessary characteristic of a blood which gives a positive F.G.T. 
In contrast to the formol-gel test and in similarity to the suspension stability test, the calcium chloride heat coagulation

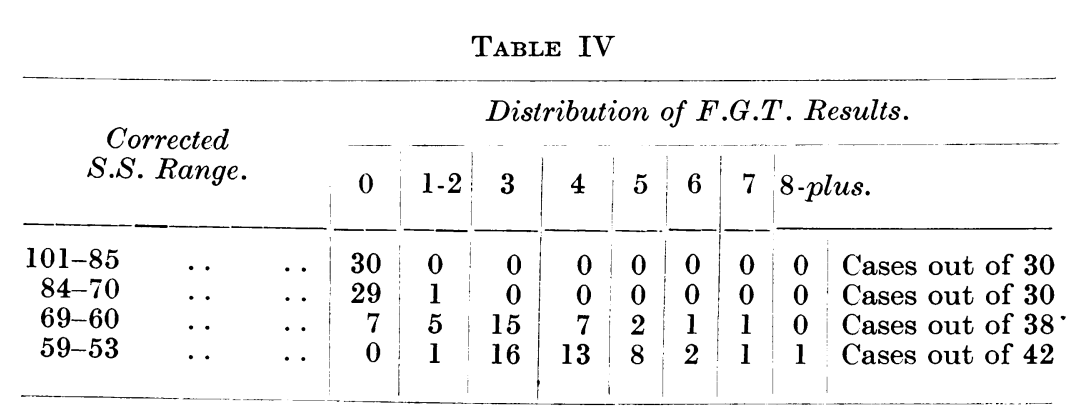

TABLE V

\begin{tabular}{|c|c|c|c|c|c|c|c|}
\hline \multicolumn{4}{|c|}{ No. of Cases. } & $\begin{array}{c}F . G . T . \\
\text { 3-plus }\end{array}$ & $\begin{array}{c}\text { Average } \\
\text { Crude } \\
\text { S.S. } \\
\\
58\end{array}$ & $\begin{array}{c}\begin{array}{c}\text { Average } \\
\text { Corrected } \\
\text { S.S. }\end{array} \\
61\end{array}$ & $\begin{array}{l}\text { Average } \\
P . C . V .\end{array}$ \\
\hline $\begin{array}{r}31 \\
20 \\
10 \\
3 \\
2 \\
1\end{array}$ & $\begin{array}{l}\ldots \\
\ldots \\
\ldots \\
\ldots \\
\ldots\end{array}$ & $\begin{array}{l}\cdots \\
\cdots \\
\cdots \\
\cdots\end{array}$ & $\begin{array}{l}\ldots \\
\ldots \\
\ldots \\
\ldots \\
\ldots\end{array}$ & $\begin{array}{l}\text { 3-plus } \\
\text { 4-plus } \\
\text { 5-plus } \\
\text { 6-plus } \\
\text { 7-plus } \\
\text { 8-plus }\end{array}$ & $\begin{array}{l}\mathbf{5 8} \\
\mathbf{5 0} \\
\mathbf{5 1} \\
\mathbf{4 7} \\
\mathbf{4 5} \\
\mathbf{4 4}\end{array}$ & $\begin{array}{l}61 \\
58 \\
58 \\
60 \\
\mathbf{5 9} \\
\mathbf{5 8}\end{array}$ & $\begin{array}{l}41 \\
\mathbf{3 7} \\
\mathbf{3 8} \\
\mathbf{3 4} \\
\mathbf{3 3} \\
\mathbf{3 3}\end{array}$ \\
\hline
\end{tabular}

reaction appears to be very sensitive, showing a fairly wide range of values for the serum coagulation band in each of the S.S. groups. These values are summarised in Table VI:

\begin{tabular}{|c|c|c|c|c|c|c|c|c|c|c|c|c|c|c|}
\hline \multirow[b]{3}{*}{$\begin{array}{c}\text { Corrected } \\
\text { S.S. } \\
\text { Range. }\end{array}$} & \multicolumn{14}{|c|}{ TABLE VI } \\
\hline & \multicolumn{14}{|c|}{ Distribution of S.C.B. Values. } \\
\hline & $\begin{array}{l}\text { Average } \\
\text { S.C.B. } \\
\text { Value. }\end{array}$ & $\begin{array}{l}0 \\
0 \\
\frac{1}{2} \\
1\end{array}$ & $\begin{array}{l}1 \frac{1}{2} \\
2 \\
2 \frac{1}{2}\end{array}$ & 3 & $3 \frac{1}{2}$ & 4 & $4 \frac{1}{2}$ & 5 & $5 \frac{1}{2}$ & 6 & $6 \frac{1}{2}$ & 7 & $\left.\begin{array}{l}7 \frac{1}{2} \\
8 \\
8 \frac{1}{2}\end{array}\right\}$ & $=S . C . B$ \\
\hline $\begin{aligned} 101-85 & \ldots \\
84-70 & \ldots \\
69-60 & \ldots \\
59-53 & \ldots\end{aligned}$ & $\begin{array}{l}6 \frac{1}{2} \\
6 \\
6 \\
5 \frac{1}{2}\end{array}$ & 1 & $\begin{array}{l}1 \\
1\end{array}$ & 3 & 0 & $\begin{array}{l}3 \\
1\end{array}$ & $\begin{array}{l}4 \\
3 \\
2\end{array}$ & $\begin{array}{r}2 \\
3 \\
2 \\
10\end{array}$ & $\begin{array}{l}2 \\
2 \\
7 \\
8\end{array}$ & $\begin{array}{r}10 \\
4 \\
8 \\
6\end{array}$ & $\begin{array}{l}6 \\
7 \\
8 \\
5\end{array}$ & $\begin{array}{l}5 \\
7 \\
4 \\
4\end{array}$ & $\begin{array}{l}5 \mathrm{ca} \\
3 \\
2 \\
1\end{array}$ & $\begin{array}{l}\text { ases } \\
\text { ", } \\
, "\end{array}$ \\
\hline
\end{tabular}

Serum coagulation band values outside the range of six to seven are regarded as showing a "shift to the left or to the right." It must be recognised clearly that a shift to the left indicates an 
exudative condition, while a shift to the right indicates a fibrotic process, but that the occurrence of both pathological changes together may result in an S.C.B. value of six to seven, indistinguishable from a result given by a healthy serum. Thus all values of six to seven are not necessarily normal. One can therefore rely only upon abnormal S.C.B. values for information about pathological conditions. The table indicates that when the S.S. is high few of the S.C.B. values show deviation to the left or right. As the S.S. becomes progressively lower, there are even fewer deviations to the right but steadily more deviations to the left. However, a close inspection of individual results in Tables I, II, IIIA, and IIIB makes it apparent that an exact parallel between S.C.B. and S.S. cannot be found.

Studying the S.C.B. results obtained at different levels of cell volume, it appears that P.C.V. variations have no influence on the serum coagulation results. Table VII indicates the relationships:

Table VII

\begin{tabular}{|c|c|c|c|c|c|c|c|c|c|}
\hline \multirow{2}{*}{$\begin{array}{l}\text { Range of } \\
\text { P.C.V. }\end{array}$} & & \multicolumn{2}{|c|}{$\begin{array}{c}\text { From } \\
\text { Table I. }\end{array}$} & \multicolumn{2}{|c|}{$\begin{array}{c}\text { From } \\
\text { Table II. }\end{array}$} & \multicolumn{2}{|c|}{$\begin{array}{c}\text { From } \\
\text { Table } I I I_{A} .\end{array}$} & \multicolumn{2}{|c|}{$\begin{array}{c}\text { From } \\
\text { Table III } .\end{array}$} \\
\hline & & $\begin{array}{l}\text { Mean } \\
\text { S.C.B. }\end{array}$ & $\begin{array}{l}\text { No. of } \\
\text { Cases. }\end{array}$ & $\begin{array}{l}\text { Mean } \\
\text { S.C.B. }\end{array}$ & $\begin{array}{l}\text { No. of } \\
\text { Cases. }\end{array}$ & $\begin{array}{l}\text { Mean } \\
\text { S.C.B. }\end{array}$ & $\begin{array}{l}\text { No. of } \\
\text { Cases. }\end{array}$ & $\begin{array}{l}\text { Mean } \\
\text { S.C.B. }\end{array}$ & $\begin{array}{l}\text { No. of } \\
\text { Cases. }\end{array}$ \\
\hline $\begin{array}{l}26-30 \\
31-35 \\
36-40 \\
41-45 \\
46-50\end{array}$ & $\begin{array}{l}\cdots \\
\cdots \\
\cdots \\
\cdots\end{array}$ & $\begin{array}{l}7 \\
7 \frac{1}{2} \\
6 \\
6 \frac{1}{2} \\
6 \frac{1}{2}\end{array}$ & $\begin{array}{r}1 \\
1 \\
8 \\
14 \\
6\end{array}$ & $\begin{array}{l}\overline{7} \\
6 \\
6 \\
6 \frac{1}{2}\end{array}$ & $\begin{array}{r}0 \\
2 \\
11 \\
15 \\
2\end{array}$ & $\begin{array}{l}6 \\
5 \\
6 \\
6 \\
6\end{array}$ & $\begin{array}{r}2 \\
5 \\
11 \\
17 \\
3\end{array}$ & $\begin{array}{l}5 \\
5 \\
5 \\
6 \\
5\end{array}$ & $\begin{array}{r}1 \\
10 \\
16 \\
14 \\
1\end{array}$ \\
\hline
\end{tabular}

In this respect, therefore, the calcium chloride heat coagulation test differs from the formol-gel test. The mean S.C.B. values shown in the table again suggest a general correlation with the S.S. results, for the S.C.B. values corresponding to the high stability bloods are seen to fall within the normal range of six to seven, while those corresponding to the low-stability bloods exhibit lower values.

The high globulin content and low albumin-globulin ratio often found in blood of low suspension stability had suggested the possibility that a number of positive mercuric chloride flocculation tests would be discovered in this series. This expectation was realised, there being complete absence of positive tests from bloods of S.S. greater than seventy, but sixteen positive 
results where the S.S. was below seventy. The occurrence of these positive reactions is shown in Table VIII:

\section{TABLE VIII}

\begin{tabular}{|c|c|c|c|c|c|}
\hline \multirow{2}{*}{$\begin{array}{l}\text { Corrected } \\
\text { S.S. Range. }\end{array}$} & \multicolumn{5}{|c|}{$\begin{array}{c}\text { Distribution of Mercuric Chloride Flocculation } \\
\text { Reactions. }\end{array}$} \\
\hline & Negative. & 1-plus. & 2-plus. & 3-plus. & Reaction Result. \\
\hline $\begin{array}{r}101-85 \\
84-70 \\
69-60 \\
59-53\end{array}$ & $\begin{array}{l}30 \\
30 \\
28 \\
36\end{array}$ & $\begin{array}{l}0 \\
0 \\
8 \\
4\end{array}$ & $\begin{array}{l}0 \\
0 \\
2 \\
1\end{array}$ & $\begin{array}{l}0 \\
0 \\
0 \\
1\end{array}$ & $\begin{array}{l}\text { Cases out of } 30 \\
\text { Cases out of } 30 \\
\text { Cases out of } 38 \\
\text { Cases out of } 42\end{array}$ \\
\hline
\end{tabular}

The incidence of positive reactions is rather low, and of the positive tests the majority were only weakly positive. Nevertheless the fact was established that bloods which yielded these positive results could thus be differentiated from other bloods of low suspension stability which failed to give positive flocculations.

When the positive flocculation tests are compared with the formol-gel results (Table IX) it appears that, with only one exception, the formol-gel tests were also positive, often to a high degree:

TABLE IX

\begin{tabular}{|c|c|c|c|c|c|c|c|c|c|c|}
\hline \multirow{2}{*}{$\begin{array}{l}\text { No. of } \\
\text { Cases. }\end{array}$} & & \multirow{2}{*}{$\begin{array}{l}\text { Mercuric } \\
\text { Chloride } \\
\text { Flocculation } \\
\text { Result. }\end{array}$} & \multicolumn{8}{|c|}{ Distribution of F.G.T. Results. } \\
\hline & & & 0 & $1-2$ & 3 & 4 & 5 & 6 & 7 & 8-plus. \\
\hline $\begin{array}{r}12 \\
3 \\
1\end{array}$ & $\begin{array}{l}\ldots \\
\cdots\end{array}$ & $\begin{array}{l}\text { 1-plus } \\
\text { 2-plus } \\
\text { 3-plus }\end{array}$ & $\begin{array}{l}1 \\
0 \\
0\end{array}$ & $\begin{array}{l}1 \\
1 \\
0\end{array}$ & $\begin{array}{l}4 \\
0 \\
0\end{array}$ & $\begin{array}{l}3 \\
0 \\
0\end{array}$ & $\begin{array}{l}2 \\
1 \\
0\end{array}$ & $\begin{array}{l}0 \\
0 \\
0\end{array}$ & $\begin{array}{l}1 \\
1 \\
0\end{array}$ & $\begin{array}{l}0 \text { cases } \\
0 \quad, \\
1 \quad,\end{array}$ \\
\hline
\end{tabular}

A similar comparison with the calcium chloride heat coagulation test (Table $\mathrm{X}$ ) reveals that only about half the bloods which gave positive flocculation yielded also abnormal serum coagulation bands, but that these were always deviated to the left:

Table $\mathrm{X}$

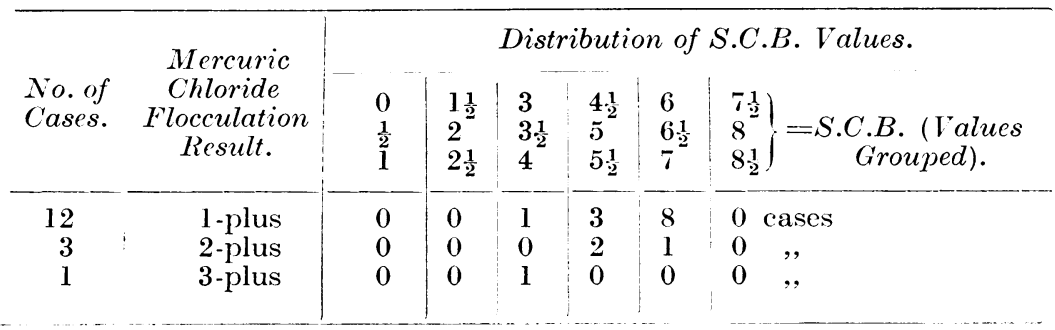


Moreover, it was found that the P.C.V. was frequently reduced below normal in those bloods which showed positive mercuric chloride flocculations (Table XI). This finding recalls a similar correlation between highly positive formol-gel tests and low cell volumes, and suggests that there is something in common between the phenomena of gelation of plasma with formalin and flocculation with mercuric chloride:

TABle XI

\begin{tabular}{|c|c|c|c|c|c|}
\hline \multirow{2}{*}{$\begin{array}{l}\text { No. of } \\
\text { Cases. }\end{array}$} & \multirow{2}{*}{$\begin{array}{c}\text { Mercuric } \\
\text { Chloride } \\
\text { Flocculation. }\end{array}$} & \multicolumn{4}{|c|}{ Distribution of P.C.V. Values. } \\
\hline & & $31-35$ & $36-40$ & $41-45$ & $46-50$ \\
\hline $\begin{array}{r}12 \\
3 \\
1\end{array}$ & $\begin{array}{l}\text { 1-plus } \\
\text { 2-plus } \\
\text { 3-plus }\end{array}$ & $\begin{array}{l}4 \\
1 \\
1\end{array}$ & $\begin{array}{l}5 \\
1 \\
1\end{array}$ & $\begin{array}{l}3 \\
0 \\
0\end{array}$ & $\begin{array}{l}0 \text { cases } \\
1 \\
0 \Rightarrow\end{array}$ \\
\hline
\end{tabular}

Finally, the relationships between the formol-gel reactions and serum coagulation bands were studied to reveal any correlation. It was found (Table XII) that the majority of bloods with negative formol-gel reactions had serum coagulation bands falling with the normal range. As the F.G.T. became progressively more strongly positive, a progressively greater proportion of shortened coagulation bands was found, while the few lengthened coagulation bands shown by the formol-gel negative bloods disappeared entirely:

TABLE XII

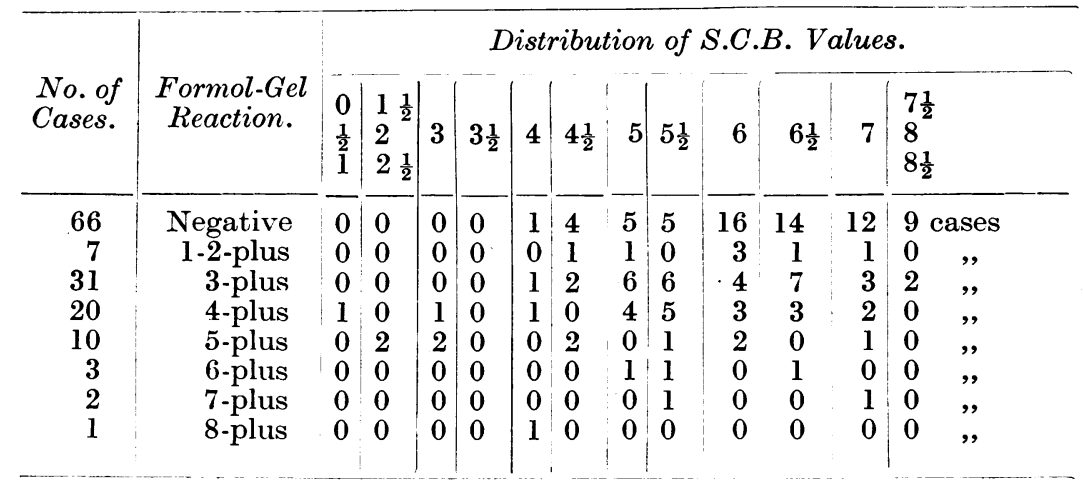

So far, the results have been discussed with little or no reference to the clinical conditions of the subjects from whom the blood specimens were obtained. None of the tests selected for study is specific for the rheumatic state, so that there is little 
chance of any definite correlation between the results of these tests and the clinical subdivisions of rheumatic disease. Moreover, the experimental studies were not especially directed towards an investigation of this aspect of the problem. Nevertheless, certain comments seem to be justified.

The seven completely negative formol-gel reactions found in the S.S. Group 69 to 70 (Table IIIA) were derived from five cases of rheumatoid arthritis of only short duration (all under nine months) in which the clinical symptoms were so indefinite as to render diagnosis doubtful; one case of mild rheumatoid arthritis previously treated with gold injections, leaving the patient symptom-free for two years; and one case of fifteen years' history of rheumatoid arthritis, clinically severe and still active, although three courses of gold treatment had been given. In this last case the negative formol-gel test was confirmed by repetition, and the result is exceptional. In the other cases the negative gel tests correspond closely with the observed clinical conditions, and appear to modify the conclusions concerning activity of the disease, which the suspension stabilities alone indicate.

Gibson and Richardson ${ }^{7}$ found that some cases of gout gave weaker formol-gel reactions than the suspension stabilities warranted. In the present series, seven cases diagnosed clinically as gout are recorded, but only one showing unusual results (Case 156, Table IIIв) need be mentioned, in which the hæmoglobin was $8 \cdot 6$ grammes per cent.; plasma uric acid, $7 \cdot 44 \mathrm{mg}$. per $100 \mathrm{ml}$; ; whole blood uric acid, 6.00 mg. per $100 \mathrm{ml}$.; and F.G.T. only 3-plus; though the P.C.V. was only 30 per cent. and the corrected S.S. 57 per cent.

Observations of the results of the calcium chloride heat coagulation test in rheumatism have not yet been made sufficiently for their significance to be recognised clearly. In the present series, noting the extreme values first, exceptionally short serum coagulation bands were found in the acute stage of rheumatic fever (Cases 116 and 88, Table IIIB), corresponding to very low suspension stabilities and highly positive formol-gel tests. A third case of rheumatic fever (No. 151, Table II), with a much greater stability and negative formol-gel test, gave an S.C.B. in the normal range corresponding with a clinically mild condition of four weeks' duration, without heart involvement. Another case (No. 102, Table IIIA), at first thought to be rheumatic but afterwards believed to be a meningococcal septicæmia 
which responded to M. and B. 693, also yielded a very low S.C.B., though the S.S. was reduced to only sixty-two. Of the three S.C.B. values of three, two were given by severe cases of rheumatoid arthritis and one by a case of toxic fibrositis, having also some rheumatoid arthritis of the knees.

The remaining S.C.B. values are best studied in Table XIII, in which the results are summarised with reference to the clinical grouping of the cases. A classification of rheumatoid arthritis in this table has been made as follows: Group 1 includes chronic rheumatoids with good general health and without any recent exacerbation of joint symptoms. Group 2 represents sub-acute rheumatoids showing recent activity in the joints and some swelling but no œdema, with fair general health. Group 3 refers to acute rheumatoid arthritis or an acute phase in a chronic rheumatoid arthritis, with painful swollen joints and odema and poor general health. Some of the rheumatoid cases were definite, but not grouped according to this plan, while others were so vague as to render the diagnosis doubtful.

Table XIII shows that the Group 2 rheumatoids and the other definite cases (ungrouped) often show a shift to the left in the S.C.B., and that this finding is even more definite in the Group 3 rheumatoids. However, other clinical types also show a shift to the left sometimes, while some of the rheumatoids, especially the less severe cases, show a shift to the right. Reference to Table VI shows that none of these deviations is very extreme in extent, excepting the six cases already noted with serum coagulation bands between 0 and 3 . It appears, therefore, that rheumatoid arthritis, particularly the severe forms of the disease, reveals itself as an exudative process by showing a moderate shift to the left in the coagulation band as determined by the calcium chloride heat coagulation test. Where cases of rheumatoid arthritis show a shift to the right in the coagulation band, it would seem that the condition is less severe and possibly has a better prognosis.

Referring, finally, to Table XIII, it is seen that the mercuric chloride flocculation test yields positive results in various clinical conditions, and it is presumed, therefore, that the reaction is indicative of plasma changes of a fundamental but non-specific nature. Of the sixteen positive reactions, only four were strongly positive. These were given by three cases of severe rheumatoid arthritis and one case of fibrositis. The remaining twelve 
positive reactions were given by nine rheumatoids, one fibrositis, one osteo-arthritis, and one case of muscular stiffness which had followed rheumatic fever for three years. At present, the exact difference between flocculation-positive and flocculation-negative bloods from cases otherwise very similar remains obscure.

TABLE XIII

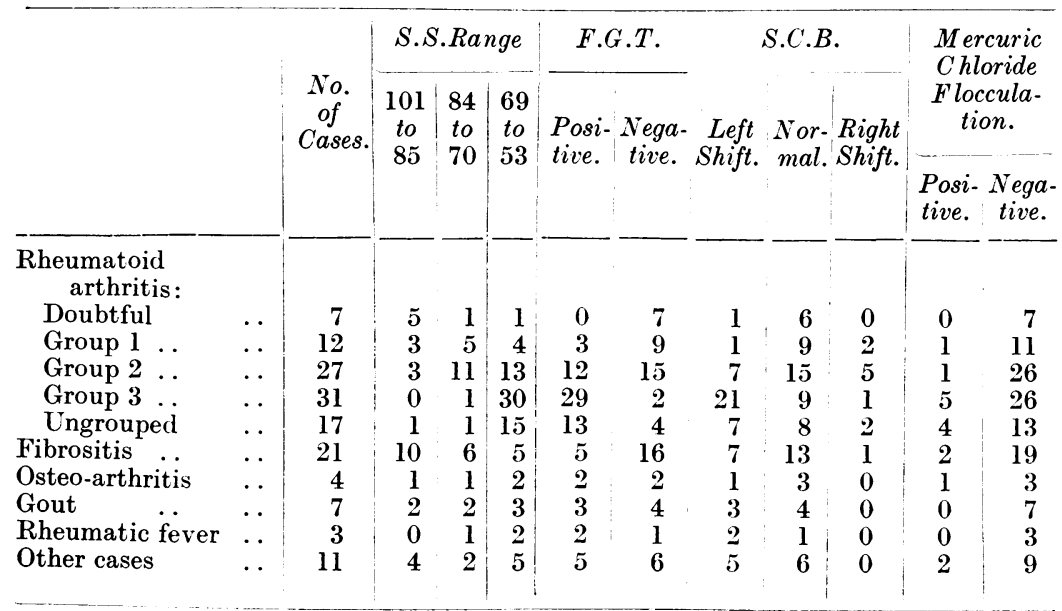

\section{Summary and Conclusions}

The use of non-specific tests for abnormal colloidal states in the blood is discussed. Four tests are described which were selected for an investigation of the blood in 140 cases of rheumatism or allied disease-namely, the suspension stability test, the formol-gel test, the calcium chloride heat coagulation test (Weltmann reaction), and the mercuric chloride flocculation test (Takata-Ara reaction). The mechanism of the tests and the significance of the results obtained therefrom are indicated.

The results of the blood tests are tabulated and discussed. The formol-gel test was almost always negative whenever the corrected suspension stability was above seventy. Negative formol-gel tests were also given by a few cases of early mild rheumatoid arthritis, although the suspension stabilities were in the range sixty-nine to sixty. Positive formol-gel tests of varying intensity were most usually obtained with bloods where the suspension stability was below seventy. Anæmic states, 
revealed by diminished packed cell volume, accounted for the occurrence of more intense gel tests than the corrected suspension stabilities would seem to predict in such cases.

The calcium chloride heat coagulation test was found to yield substantially normal serum coagulation bands whenever the corrected suspension stability was high. As the stabilities progressively diminished through the series, the serum coagulation bands also diminished-i.e., became deviated to the left-though an exact parallel between the suspension stability and the serum coagulation band could not be detected in every individual case. Variations in volume of packed cells had no apparent influence on the value of the serum coagulation bands. Negative formolgel tests were usually associated with normal coagulation bands, while progressively more intense gel reactions were found to be accompanied by a progressive shift to the left in the serum coagulation band.

The mercuric chloride flocculation test was invariably negative whenever the corrected suspension stability was greater than seventy. Among eighty blood specimens of stability below seventy, sixteen positive flocculation tests were discovered. According to expectations, these sixteen bloods also showed positive formol-gel tests in almost every case, but showed abnormal serum coagulation bands in only about half the cases. The abnormal coagulation bands were always shortened-i.e., deviated to the left. A reduction in the volume of packed cells was frequently associated with positive mercuric chloride flocculation reactions.

Comparing the results of the blood tests with the clinical conditions of the patients, it appears that both the suspension stability test and the calcium chloride heat coagulation test are very sensitive indicators of the extent of activity of rheumatic disease. But the two tests do not always run parallel. The significance of the serum coagulation band in rheumatism is not clearly understood, and extensive observations on this point should be made, as they would probably render the calcium chloride heat coagulation test as useful to the rheumatologist as is the suspension stability test. Already it has been found that severe rheumatoid arthritis is characterised by a moderate shift to the left in the serum coagulation band, and that rheumatic fever in the acute stage produces a much greater deviation to the left. An occasional shift to the right was found 
in rheumatoid arthritis, and this would seem to be a sign of good prognosis. However, further observations are clearly desirable.

The usefulness of the formol-gel test is restricted to a confirmation of the significance of low suspension stabilities. Strongly positive gel-tests reinforce the meaning of the low stabilities, and are often accompanied by low packed cell volumes, while occasional weakly positive or negative gel tests may modify the significance attributed to low stabilities.

Positive mercuric chloride flocculation tests are occasionally to be found where suspension stabilities are low, especially where the formol-gel test is strongly positive and volume of packed cells diminished. While such results are most frequently found in rheumatoid arthritis, the exact meaning of the positive flocculation tests remains obscure, and awaits elucidation by further observations relating clinical states with the mercuric chloride test.

\section{REFERENCES}

1. RACE, J. (1935): Rep. Chron. Rheum. Dis., 1, 55.

2. Davis, J. S., JR. (1936): Journ. Lab. and Clin. Med., 21, 478.

3. Scull, C. W., Bach, T. F., and Pemberton, R. (1939): Ann. Int Med., 12, 1463 .

4. Ropes, M. W., Rossmeisl, E., and Bauer, W. (1939): Journ. Clin. Invest., 18, 791.

5. Davison, R. (1940): Journ. Lab. and Clin. Med., 25, 935.

6. Colinns, D. H., Gibson, H. J., RaCe, J., and Salt, H. B. (1939): Ann. Rheum. Dis., 1, 333.

7. Gibson, H. J., ANd Richardson, E. W. (1938): Acta Rheumatologica, 10, 30.

8. Green, C. A., Thomson, S., and Glazebrook, A. J. (1939): Ann. Rheum. Dis., 1, 180.

9. WeltmanN, O. (1930): Med. Klin., 26, 240.

10. TeUfL, R. (1936): Wiener Archiv. für Innere Med., 29, 37, 297.

11. Hennes, H., AND Kemen, A. (1936): Klin. Wochenschrift, 15, 378.

12. Levinson, S. A., Klein, R. I., ANd Rosenblum, P. (1937): Journ. Lab. and Clin. Med., 23, 53.

13 Levinson, S. A., And Klein, R. I. (1938): Amer. Rev. Tuberc., 37, 200.

14. Levinson, S. A., ANd Klein, R. I. (1939): Ann. Int. Med., 12, 1948.

15. Klein, R. I., Levinson, S. A., ANd Rosenblum, P. (1940): Amer. Journ. Dis. Child., 59, 48.

16. Crane, M. P. (1934): Amer. Journ. Med. Sci., 187, 705.

17. KIrK, R. C. (1936): Journ. Amer. Med. Assoc., 10\%, 1354.

18. Bowman, R. O., and Bray, R. S. (1937): Journ. Lab. and Clin. Med., 22, 532 . 
19. Chasnoff, J., And Solomon, S. (1938): Journ. Lab. and Clin. Med., 23, 887, 894 .

20. Taran, A., and Lipstein, S. (1939): Journ. Lab. and Clin. Med., 24, 479 .

\section{OBSERVATIONS ON RHEUMATIC DISEASES UNDER WAR CONDITIONS*}

\section{By OSWALD SAVAGE}

Since the outbreak of the German air offensive against this country an opportunity has arisen to observe patients in the various stages of rheumatic diseases who have been living under active war conditions.

The intermittent bombing of London which for long spells has been of nightly occurrence has brought both mental and physical stress which would tend to cause the onset of rheumatic conditions in those susceptible and the aggravation of the symptoms of those already suffering.

The attendance at both clinics where I have worked has shown an increase during the last year despite the large evacuation from London and the fact that many of those requiring treatment have come irregularly owing to the difficulties of travel and the fear that the clinic might have been destroyed. The only decrease has been in the type of patient with rather vague rheumatic pains and no clinical abnormality who responded to many types of treatment for a time and then passed on to another clinic. These were usually of a nervous temperament and may have taken advantage of the evacuation schemes.

The feature most directly affecting the health of Londoners is the necessity of the nights spent in shelters. The two most detrimental elements are the shortage of sleep and the cold and damp often prevalent. Rheumatic subjects are particularly vulnerable.

I have questioned patients on the types of shelters they use and have seen representative examples.

In the suburbs the Anderson shelter is most common, and as these shelters are sunk into the ground it is impossible to exclude

\footnotetext{
* Received for publication March 24, 1941.
} 